\title{
Modelling of the populating development of the genome in the radiation condition of the environment
}

\author{
Andrey N. Volobuev*, Eugene S. Petrov
}

Samara State Medical University, Samara, Russia; *Corresponding Author: volobuev@samaramail.ru

Received 8 November 2011; revised 30 November 2011; accepted 12 December 2011.

\begin{abstract}
The existence of the genome population in condition of radiation environment has been considered. The differences between the laws of the allele frequencies for autosomal genes and genes linked to sex are described. Radiation conditions were found at maintenance of the balance of the Hardy-Weinberg genotype in the population, as well as conditions of complete elimination of the targeted allele by ionizing radiation. Conclusions about the nature of radiation resistance of the population are drawn.
\end{abstract}

Keywords: Population; lonizing Radiation; Genotypes; Frequency of Allele; Recessive Allele; Heterozygote; Hardy-Weinberg Equilibrium; Inbreeding

\section{INTRODUCTION}

Intensive production, extracting and use of radioactive elements, the lack of protection system and so on, as well as accidents at nuclear power plants - all these reasons lead to the fact that elevated background radiation is a source of mutations affecting the gene pool of the human population, which may cause irreversible consequences not only for living people, but also for future generations [1-4]. It is therefore interesting to consider the existence and development of the population in exposure to mutagenic ionizing radiation.

\section{HARDY-WEINBERG EQUILIBRIUM}

When crossed opposite-sex individuals in one generation, equilibrium frequency of alleles is established for the autosomal genome, the law of which was found by the English mathematician Hardy and German physician Weinberg in 1908 - the so-called Hardy-Weinberg equilibrium.

Under this law, the genotypes $A A, A a$, and $a a$ have the following frequency relation:

$$
(A A) p^{2}:(A a) 2 p q:(a a) q^{2}
$$

where $p$-is the dominant frequency of allele $A, q$ - the frequency of the recessive allele $a,(p+q=1)$ so that

$$
\text { (A) } p:(a) q
$$

Ratio (1) remains unchanged from generation to generation in an ideal population (number of individuals is very large, there is pan-mixing, there is no selection, mutation, migration of individuals, etc.) [5]. Violation ratio (1) due to any external influences: mutation, migration, and so on-leads only to a change in the next generation of gene frequency ratio (2) and recovery ratio (1).

One of the most significant factors of mutagenesis is ionizing radiation. Violations of the genetic apparatus, arisen with it, are generally incompatible with the normal functioning of the organism further. Mutations in genes in sex chromosomes can lead either to the disappearance of reproductive function in individuals, or to the appearance in the offspring of serious violation.

We will analyze the existence of a population in a radiation environment, and mathematical modeling of changes in the gene pool taking as an example changes in both sex-linked allele frequencies and inherited autosomally.

This problem can be solved analytically in complete form.

Assume that during the impact on the population, the frequencies of genotypes changed in the following proportions:

$$
\begin{aligned}
(A A)\left(p^{2}+F p q+\alpha\right) & :(A a)[2 p q(1-F)+\beta] \\
& :(a a)\left(q^{2}+F p q+\gamma\right)
\end{aligned}
$$

that breaks the Hardy-Weinberg relation (1) (or taking into account to inbreeding-Wright). In (3) condition of pan-mixing weakened. The opportunity of closely-related marriages with inbreeding coefficient $F$ there are. This ratio characterizes the decrease in the proportion of individuals in heterozygote $(1-F)$ times compared with 
pan-mixed population.

In reality, the impact on the population may be related to migration, the emergence of subpopulations, radiation exposure, negative social phenomena, etc.

In the next generation Hardy-Weinberg equilibrium is restored with the new correlation of allele frequencies. To obtain the dominant frequency of allele $A$ in the new balance must sum up the frequency of homozygote $A A$ and half the frequency of heterozygote $A a$ from (3). Similarly we will do and for the allele $a$. As a result, we obtain

$$
(A)(p+\alpha+\beta / 2):(a)(q+\gamma+\beta / 2)
$$

Thus, the ratio of the frequencies of genotypes in the next generation is

$$
\begin{aligned}
& (A A)(p+\alpha+\beta / 2)^{2} \\
& :(A a) 2(p+\alpha+\beta / 2) \cdot(q+\gamma+\beta / 2) \\
& :(a a)(q+\gamma+\beta / 2)^{2}
\end{aligned}
$$

Let's note that in (5) and (4) is not an inbreeding coefficient $F$.

Let us consider some consequences of the resulting frequency ratios (4) and (5).

In the absence of influences on the population, i.e. $\alpha=\beta=\gamma=0$, ratio (5) is identical to (1), i.e. we have a well-known position to maintain the frequency of genotypes in a set of generations.

When exposed to mutagenic agents on the population of a differently nature Hardy-Weinberg equilibrium is restored, but the population does not compensate for the impact - the allele frequencies changed. This gives an answer to the question of what type of equilibrium takes place: stable, unstable and indifferent. Hardy-Weinberg equilibrium is indifferent.

The mechanical analogy of the three possible types of equilibrium: stable-1, unstable-2, indifferent-3, shown in Figure 1.

\section{INFLUENCE ON A POPULATION OF RADIOACTIVE RADIATION}

The question of the long and gradual reduction of the
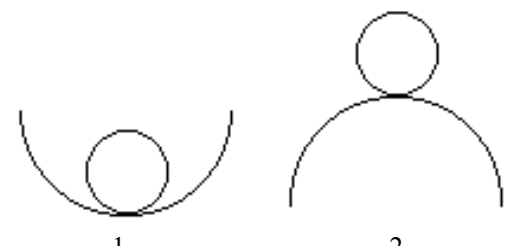

2

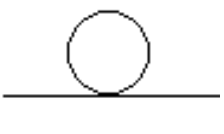

Figure 1. Possible types of equilibrium (1: stable, 2: unstable, 3: indifferent). Mechanical analogy of the Hardy-Weinberg equilibrium. radiation exposure in which the population evolves, is of a great interest.

Let's consider a model situation where the existence of a population, in the region it is only one element of the radioactive half-life $T$. $A$ was established [6] that the frequency of mutations (usually lethal) is proportional to the power dose of radiation $p=k_{1} P$, where $k_{1}$ is a constant. As the power dose of radiation is proportional to the activity of radioactive elements, scattered in the environment, it is possible to record the frequency of mutations, using the fundamental law of radioactive decay, the ratio $p \sim\left(\frac{1}{2}\right)^{\left(\frac{t}{T}\right)^{n}}$, where $t \sim 30$ years is the approximate time of one generation [6], $n$ is a number of the considered generation the existing in conditions of radiation.

$$
\text { Accepting } R=\left(\frac{1}{2}\right)^{\left(\frac{t}{T}\right)} \text { and considering the influence }
$$
of radiation on recessive homozygote only aa, i.e. $p=\gamma+\beta / 2$, we obtain:

$$
\gamma+\beta / 2=(k / 2) R^{n}
$$

where the coefficient $1 / 2$ is taken for the convenience of further transformations. The coefficient $k$ characterizes the initial activity of the radioactive elements that affect the population. In accordance with [7] $k \sim N_{0} / T$, where $N_{0}$ is the total initial quantity of a radioactive substance that acts on a population.

For example, there are recessive genes responsible for the content of catalase in the blood. One can cite as an example of reducing the frequency of recessive alleles of these genes at 15.5 times for about two generations of people living in Hiroshima and Nagasaki in comparison with the general population of Japan. In this case, the frequency of dominant alleles has not changed [8].

Based on (4), the recursive formula for changing the frequency of allele $a$ for the generations of the population is as follows:

$$
q_{n}=q_{n-1}=+\gamma+\beta / 2=q_{n-1}+(k / 2) R^{n}
$$

where $k<0, R<1 \quad$ (at $t=T, R=\frac{1}{2}$ ).

The solution of this equation is not difficult and it is the sum of a geometric progression:

$$
q_{n}=q_{0}+\frac{k}{2} \frac{R\left(1-R^{n}\right)}{1-R}
$$

where $q_{0}$-is the frequency of allele $a$ in the initial generation.

If the number of generations tends to infinity $n \rightarrow \infty$, frequency of allele $a$, tends to the value: 


$$
\hat{q}=q_{0}+\frac{k}{2} \frac{R}{1-R} .
$$

The possibility of establishing equilibrium is determined by the condition $q_{0}>\left|\frac{k}{2} \frac{R}{(1-R)}\right|$. Taking $q_{0}=0$, we can solve Eq.8 with respect to $n$ a number of generations for which will complete elimination of the allele $a$ that reduces the variability of the population's gene pool.

Figure 2 is a graph of the dependence frequency $q_{n}$ of allele $a$ the number of generations $n$, constructed in accordance with the Formula (8) for the half-life of radioactive elements in the region, respectively, $T=176$; 93.8 and 22.6 years. For example, half-life of radioactive substances is such as ${ }_{55} \mathrm{Cs}^{137}-30.2$ years, ${ }_{38} \mathrm{Sr}^{90}-28.7$ years, and ${ }_{27} \mathrm{Co}^{60}-5.3$ years [9]. But usually a half-life isotope is considerably smaller.

As can be seen from the graphs, for the half-life 93.8 years the allele $a$ will be disappears in the eighth generation. For the half-life $T=22.6$ years after a fifth-generation set a new equilibrium frequency of allele $a \hat{q}=0.17$. In the calculations there are for allele $a$ used the initial frequency $q_{0}=0.9 ; k=-\frac{50}{T}$.

The critical value of the half-life, above which the Hardy-Weinberg equilibrium is not established as a result of complete elimination of the allele $a, T=37.4$ years.

Figure 2 shows that for small half-life, curve 3, the frequency of alleles $a$ at first drops very quickly, because high activity radioactive elements. But then equilibrium is established, because rapid decrease in the number of highly active substances reduces the effects of radiation. For long half-life the frequency of allele $a$ decreases more slowly, but there are completely eliminates the allele at the affect of long radiation exposure, curves 1 and 2.

Suppose that $A$ and $a$ alleles and linked to the $X$ chromosome. The frequency of allele $A$ in men and women is $p_{m}$ and $p_{f}$. For allele $a$ they will be $q_{m}$ and $q_{f}$.

When crossed in the first generation there are arise genotype correlation in women:

$$
(A A) p_{f} p_{m}:(A a)\left(p_{m} q_{f}+p_{f} q_{m}\right):(a a) q_{m} q_{f} .
$$

In men, there is a frequency ratio gemizygote determined that the $X$ chromosome in female in crossing goes to the male offspring:

$$
\text { (A) } p_{f}:(a) q_{f}
$$

Suppose, as in the case of autosomal inheritance, on the population act external factor, changing the ratio of genotype frequencies in women:

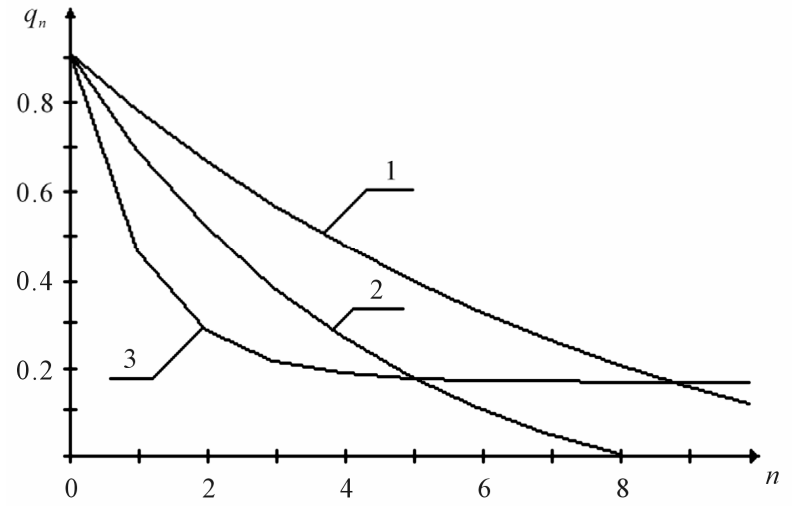

Figure 2. The relationship between the frequency $q_{n}$ affected by the radiation of the alleles of autosomal genes and the number of generations $n$ populations for different half-life (1: $T$ $=176$ years, $2: T=93.8$ years, $3: T=22.6$ years).

$$
\begin{aligned}
& (A A)\left[p_{f} p_{m}+\left(p_{m} q_{f}+p_{f} q_{m}\right) F / 2+\alpha\right] \\
& :(A a)\left[\left(p_{m} q_{f}+p_{f} q_{m}\right)(1-F)+\beta\right] \\
& :(a a)\left[q_{f} q_{m}+\left(p_{m} q_{f}+p_{f} q_{m}\right) F / 2+\gamma\right]
\end{aligned}
$$

Assume also that the gemizygote state men (11) is unaffected. Then, using the previous method of calculating allele frequencies, we can find that the change in frequency of alleles $a$ generations of women is

$$
q_{f n}=\frac{1}{2}\left[q_{f(n-1)}+q_{m(n-1)}\right]+\gamma+\beta / 2 .
$$

As in the case of autosomal inheritance, the inbreeding coefficient of $F$ in relation (13) is not included.

Take into account that the $X$-chromosome male inherits from his mother: $q_{m(n-1)}=q_{f(n-2)}$.

As in the case of autosomal inheritance, we consider the effects of ionizing radiation on the recessive homozygote $a a$.

$$
q_{f n}=\frac{1}{2}\left[q_{f(n-1)}+q_{f(n-2)}\right]+(k / 2) R^{n}
$$

Solution of this equation is the sum of the general solution of the homogeneous Eq.15 and a particular solution of inhomogeneous Eq.14 [10]:

$$
\tilde{q}_{f n}=\frac{1}{2}\left[\tilde{q}_{f(n-1)}+\tilde{q}_{f(n-2)}\right]
$$

so

$$
q_{f n}=\tilde{q}_{f n}+q_{f n}^{*} .
$$

The general solution of homogeneous Equation (15) $\tilde{q}_{f n}$ will be sought in the form $\tilde{q}_{f n}=a^{n}$. Substituting this expression into (15), we find:

$$
a^{n}=\frac{1}{2}\left[a^{n-1}+a^{n-2}\right]
$$


Dividing both sides by $a^{n-2}$, we obtain a quadratic characteristic equation:

$$
2 a^{2}-a-1=0
$$

the solution of which are the two roots $a_{1}=1 ; a_{2}=-\frac{1}{2}$.

So, the general solution of (15) has the form:

$$
\tilde{q}_{f n}=C_{1}+C_{2}\left(-\frac{1}{2}\right)^{n}
$$

where $C_{1}$ and $C_{2}$-are constants. Solution (19), with the initial conditions, describes the change of frequency allele $a$ women in the generations in the absence of external factors on the population.

A particular solution of Eq.14 will be sought in the form $q_{f n}^{*}=L R^{n}$, where $L$-is yet unknown. Substituting this formula into (14) we find:

$$
2 L R^{2}-L R^{n-1}-L R^{n-2}=k R^{n}
$$

Dividing both sides by $R^{n-2}$, we find the value of $L$ :

$$
L=\frac{k R^{n}}{2(R-1)(R+1 / 2)} \text {. }
$$

Thus, the solution of Eq.14 in accordance with (16) is:

$$
q_{f n}=C_{1}+C_{2}\left(-\frac{1}{2}\right)^{n}+\frac{k R^{n+2}}{2(R-1)(R+1 / 2)}
$$

Constants $C_{1}$ and $C_{2}$ we find the basis of the initial conditions at $n=0, q_{f n}=q_{f 0}$ and at $n=1$ according to (13) and (6)

$$
\begin{aligned}
q_{f n} & =q_{f 1}=\left(q_{m 0}+q_{f 0}\right) / 2+\gamma+\beta / 2 \\
& =\left(q_{m 0}+q_{f 0}\right) / 2+(k / 2) R
\end{aligned}
$$

Consequently, the solution of Eq.14 is:

$$
\begin{aligned}
q_{f n}= & \frac{2 q_{f 0}+q_{m 0}}{3}-\frac{k R}{3(R-1)} \\
& +\left[\frac{q_{f 0}-q_{m 0}}{3}-\frac{k R}{6(R+1 / 2)}\right]\left(-\frac{1}{2}\right)^{n} \\
& +\frac{k R^{n+2}}{2(R-1)(R+1 / 2)}
\end{aligned}
$$

If the number of generations in women tends to infinity $(n \rightarrow \infty)$, there is an equilibrium frequency of allele $a$ (if it is possible):

$$
\hat{q}_{f n}=\frac{2 q_{f 0}+q_{m 0}}{3}-\frac{k R}{3(R-1)}
$$

Similarly to the case of autosomal inheritance at $q_{f n}=0$ the number of generations, which would completely eliminate allele $a$ in women can be found.
Figure 3 is a graph of the dependence of the frequency $q_{f n}$ of allele $a$ in women and the number of generations $n$, constructed by the Formula (23) for the same half-life as for autosomal inheritance.

As can be seen from the graphs, for the half-life $T=$ 93.8 years - the allele $a$ will be disappears in the ninth generation, the curve 2. For the half-life $T=22.6$ years since the sixth generation a new equilibrium is established with the frequency of allele $a$ in women $\hat{q}_{f n}=0.14$, curve 3 . Curve 1 is designed for the half-life of $T=176$ years. At the calculations used the initial frequency of allele $a$ women $q_{f 0}=0.9$; men $q_{m 0}=0.1$.

The critical value of the half-life, above which the Hardy-Weinberg equilibrium is not established as a result of complete elimination of the allele $a$, in the case of inheritance of genes linked to the $X$ chromosome, $T=$ 44.7 years.

Figure 4 is a graph of the number of generations $n_{k}$ of the complete elimination of the allele $a$ and half-life of radioactive element $T$ for autosomal genes. 1 and the genes of sex-linked, 2. The graphs are based on the solutions of Eqs.8 and 23 at initial conditions, respectively, $q_{n}=0$ and $q_{f n}=0$.

The graphs show that there are more dangerous radioactive elements to the population. For the given model calculation they are the elements with half-life $T$ $=52.2$ years for autosomal genes and $T=60.2$ years for genes linked to sex. In the first case of complete elimination of the allele $a$ it will occur after $n_{k} \approx 6$ generations, and in the second case - after $n_{k} \approx 8$ generations. Thus, genes, sex-linked, are more resistant to radiation than autosomal.

\section{CONCLUSIONS}

As a result of calculations we can draw the following

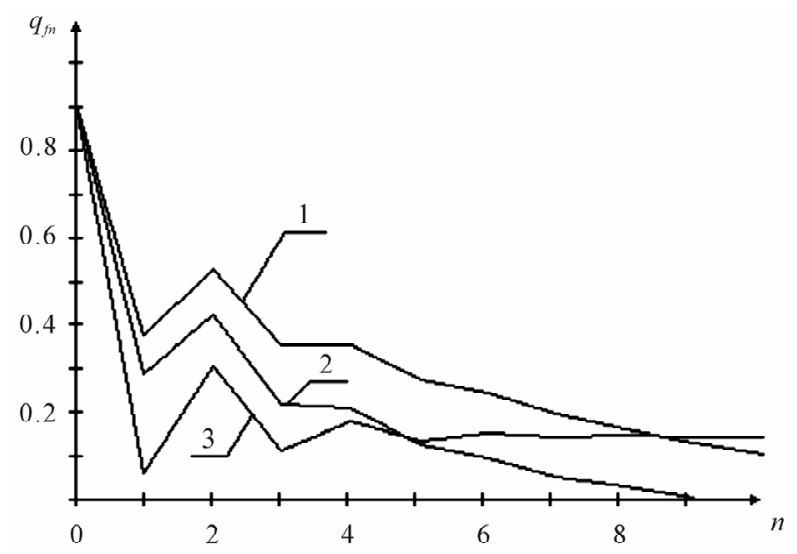

Figure 3. The relationship between the frequency $q_{f n}$ affected by the radiation of the alleles of the gene linked with $X$-chromosome in females and the number of generations $n$ populations for different half-life (1: $T=176$ years, $2: T=93.8$ years, 3: $T=22.6$ years). 


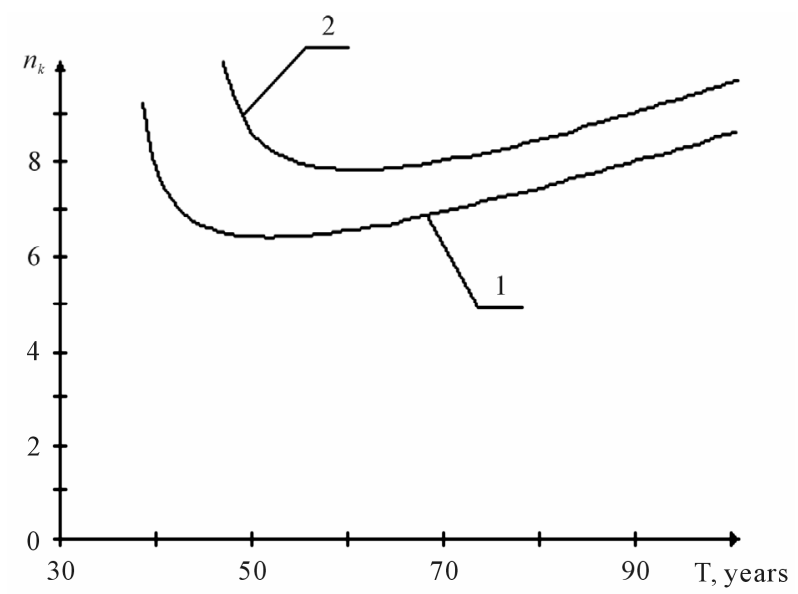

Figure 4. The relationship between the limiting number of generations $n_{k}$ population which still retained its gene pool and installed the Hardy-Weinberg equilibrium for the targeted of affected radiation gene and half-life of radioactive element $\mathrm{T}$ (1: for autosomal genes, 2: for genes linked with $X$-chromosome).

conclusions:

1) When there is the action of radiation on the population 2 outcomes are possible depending on the half-life of radioactive elements in the environment:

- Establishment of the Hardy-Weinberg equilibrium at maintaining the existing in the population genome, but at a lower level of the targeted allele frequencies;

- The disappearance of the targeted allele, and consequently the impossibility of preserving the HardyWeinberg equilibrium.

2) There is a boundary value of the half-life of radioactive substances in the environment, below which the population maintains its genome. Consequently, the outcome of the population depends on the nature of the radiation environment (disaster at nuclear power plants, nuclear explosion, accident at work, a natural phenomenon, etc.).

3) Genes that are sex-linked, have a somewhat higher resistance to radiation compared with the autosomal ones.

4) Inbreeding does not affect the radiation resistance of the population.

The obtained results allow us to be more cautious, at presence the choice, to use of radioactive elements with specific half-lives, which have a strong mutagenic effect on autosomal genes and genes linked to the $X$-chromosome.

Considering in the evolutionary terms that genes linked to the $X$-chromosome have greater resistance, we can recall that genes are located in the $X$-chromosome, mutations of which affect the vital functions of the human organism (vision, blood clotting, skin [11]). Therefore, these genes are phylogenetically were better protected against the action of mutagens such as radiation. Greater resistance to radiation of alleles, which are localized in 23 pairs of chromosomes, again suggests that the female organism is genetically more stable. It plays an important role in maintaining the size of the population.

The obtained results allow us to determine the radiation conditions of the maintaining of the balance of the Hardy-Weinberg in the population, as well as conditions of complete elimination of the targeted allele by ionizing radiation.

The above method of analysis of the effects of radiation on the genome can be applied to other types of influences. Thus it is necessary only to replace the fundamental law of radioactive decay in (6) and (14) with another one for the investigated impact.

\section{REFERENCES}

[1] Sankaranarayanaan, K. (2006) Estimation of the genetic risks of exposure to ionizing radiation in humans: Current status and emerging perspectives. Journal of Radiation Research, 47, 57-66.

[2] Yin, E., Nelson, D., Coleman, M., Peterson, L. and Wyrobek, A. (2003) Gene expression changes in mouse brain after exposure to low-dose ionizing radiation. International Journal of Radiation Biology, 79, 759-775. doi:10.1080/09553000310001610961

[3] George, K., Durante, M. and Cucinotta, F.A. (2007) Chromosome aberrations in astronauts. Advances in Space Research, 40, 483-490. doi:10.1016/j.asr.2007.03.100

[4] Brenner D.J. and Hall E.J. (2007) Computed tomography -An increasing source of radiation exposure. New England Journal of Medicine, 357, 2277-2284. doi:10.1056/NEJMra072149

[5] E. Libberta. (Ed.) (1982) Basics of general biology. Springer-Verlag, Berlin, 328.

[6] Vogel, F. and Motulsky, A. (1990) Human genetics. 2, Springer-Verlag, Berlin, 226.

[7] Yavorsky, B.M. and Dettlaff, A.A. (1990) Guide to physics. Nauka, Moscow, 525.

[8] Ayala, F. and Kiger, Jr. (1988) Modern genetics. 3, Mir, Moscow, 134, 297.

[9] Kostyuk, P.G., Grodzinsky, D.M., et al. (1988) Biophysics. Vyshcha Shkola, Kiev, 389.

[10] Godunov, S.K. and Ryaben'kii, V.S. (1977) Difference schemes. Nauka, Moscow, 29.

[11] Kozlov, S.I., Semenov, E.T., Demikova N.S., Blinnikova, O.E. (1987) Hereditary syndromes, and medical and genetic counseling. Medicine, St. Peterburg, 320. 